\title{
Correlaçóes entre a Participação Ocupacional, Independência e Cognição EM Adultos com DeficiênCIa Física ${ }^{1}$ Correlation among OcCupational PARTicipation, Independence and Cognition in Adults With Physical Disabilities
}

\author{
Daniel Marinho Cezar da CRUZ² \\ Sue PARKINSON ${ }^{3}$ \\ Débora Couto de Melo CARRIJO ${ }^{4}$ \\ Jacqueline Denubila COSTA5 \\ Emerson FACHIN-MARTINS 6 \\ Mariana Gurian MANZINI ${ }^{7}$ \\ Luzia Iara PFEIFER ${ }^{8}$
}

\begin{abstract}
RESUMO: A participação ocupacional é uma questão crucial para a atenção à pessoa com deficiência e caracteriza-se pelo grau de participação de uma pessoa em várias ocupaçôes, tais como as de autocuidado, trabalho, escola, lazer, dentre outras. O objetivo deste estudo foi, assim, verificar se a participação ocupacional avaliada pelo Model of Human Occupation Screening Tool (MOHOST Brasil) apresenta correlaçóes com as medidas de independência funcional e de cognição em adultos com deficiências. Foram avaliadas 41 pessoas com deficiência física usando o MOHOST Brasil, a Medida de Independência Funcional (MIF) e o Mini Exame do Estado Mental (MEEM). A subescala motora da MIF apresentou correlaçâo moderada com o fator de habilidades motoras MOHOST (r 0,586 p = 0,001). O item "Linguagem e práxis" do MEEM apresentou correlação moderada com o fator "habilidades de processo" do MOHOST (r 0,573 p = 0,001). As correlaçóes entre o MOHOST e a MIF e entre o MOHOST e o MEEM mostram que há relação entre as dimensões de participação, desempenho e habilidades, confirmando que o MOHOST é útil para avaliar a participação ocupacional de pessoas com deficiências físicas. Conclui-se também que o MOHOST pode fornecer dados que possam contribuir para o planejamento de intervenções que estimulem a participação ocupacional em diferentes papéis no âmbito da Terapia Ocupacional e na sua inferface com a Educação Especial.
\end{abstract}

PALAVRAS-CHAVE: Ocupaçóes. Avaliação de resultados de intervençóes terapêuticas. Prática clínica baseada em evidências. Terapia Ocupacional. Educação Especial.

ABSTRACT: Occupational participation is a crucial issue for people with disabilities care and it is characterized by the degree of a person's participation in various occupations, such as self-care, work, school, leisure, among others. Thus, this study's objective was to examine if the occupational participation measured by Model of Human Occupation Screening Tool (MOHOST Brazil)

\footnotetext{
${ }^{1}$ https://doi.org/10.1590/1980-54702021v27e0162

${ }^{2}$ Doutor em Educação Especial. Universidade Federal de São Carlos. São Carlos/São Paulo/Brasil. E-mail: cruzdmc@gmail.com. ORCID: https://orcid.org/0000-0002-4708-354X

${ }^{3}$ Terapeuta Ocupacional, Treinadora autônoma do MOHO. Chestershire/Derbyshire/Reino Unido. E-mail: spark39@hotmail. co.uk. ORCID: https://orcid.org/0000-0002-7155-505X

${ }^{4}$ Doutorado em Ciência. Universidade Federal de São Carlos. São Carlos/São Paulo/Brasil. E-mail: coutocarrijo@gmail.com. ORCID: https://orcid.org/0000-0002-2463-6690

${ }^{5}$ Mestrado em Terapia Ocupacional. Universidade Federal de São Carlos. São Carlos/São Paulo/Brasil. E-mail: jack_denubila@ hotmail.com. ORCID: https://orcid.org/0000-0001-6019-1935

${ }^{6}$ Doutorado em Neurociências e Comportamento. Universidade de Brasília, Faculdade de Ceilândia, Setor Campus Universitário Centro Metropolitano. Ceilândia Sul/Brasília/Brasil. E-mail:efmartins@unb.br. ORCID: https://orcid.org/0000-0001-9858-9009

${ }^{7}$ Doutorado em Educação Especial. Universidade Federal de São Carlos, São Paulo, Brasil. E-mail: mariana_gurian@yahoo.com. br. ORCID: https://orcid.org/0000-0002-9126-7514

${ }^{8}$ Doutorado em Educação. Universidade Federal de São Carlos. São Carlos/São Paulo/Brasil. E-mail: luziara@fmrp.usp.br. ORCID: https://orcid.org/0000-0002-1826-1968
} 
has correlations with measures of functional independence and cognition in adults with disabilities. Forty-one ( $\mathrm{n}=41)$ people with physical disabilities were assessed using the MOHOST Brazil, Functional Independence Measure (FIM) and the Mini-Mental State Examination (MMSE). The FIM's motor subscale presented moderate correlation with the MOHOST motor skills factor (r $0.586 \mathrm{p}=0.001)$. The MMSE's item “Language and praxis" presented moderate correlation with the MOHOST's factor "process skills" ( $\mathrm{r} 0.573 \mathrm{p}=0.001$ ). The correlations between the MOHOST and FIM and between the MOHOST Brazil and MMSE show there is an inter-relation between the dimensions of participation, performance and skills, confirming that the MOHOST is useful for assessing occupational participation of people with disabilities. It is also concluded that MOHOST can provide information that can contribute on planning interventions to support occupational participation in different roles for Occupational Therapy and its possibilities of work in the field of Special Education.

KEYWORDS: Occupations. Evaluation of results of therapeutic interventions. Evidence-based clinical practice. Occupational Therapy. Special Education.

\section{INTRODUÇÁo}

A participação tornou-se um conceito central nos cuidados de saúde e educação e, mais especificamente, na terapia ocupacional (Vessby \& Kjellberg, 2010). A participação ocupacional refere-se ao grau de participação de uma pessoa em várias ocupaçóes, tais como as de autocuidado, trabalho, escola, lazer, dentre outras.

Para Parkinson et al. (2006) o conceito de participação ocupacional abrange o envolvimento no trabalho, no brincar ou nas atividades da vida diária que fazem parte do contexto social das pessoas. A participação ocupacional informa os diferentes papéis ocupacionais que as pessoas autorreferem ter, por exemplo: uma criança na escola apresenta um papel como estudante, sendo, assim, expectativas sociais e de desempenho esperadas para esse papel, tais como: frequentar a escola, assistir às aulas, realizar tarefas escolares em casa, relacionar-se com os seus pares, manipular materiais escolares como lápis, borracha, apontador dentre outros. Esses exemplos informam graus de participação ocupacional em papéis. Para as pessoas com deficiência, as medidas de participação tornam-se cruciais para o planejamento de açóes que possam promover a participação dessas pessoas em ocupaçôes que sejam significativas para elas, sendo essa uma das estratégias para a sua inclusão.

As intervenções de terapia ocupacional a partir do Modelo da Ocupação Humana (MOHO) são baseadas em extensa teoria sobre os constructos: motivação, hábitos, papéis ocupacionais, habilidades (motoras, processuais, de comunicação e interação) e ambiente, para facilitar a participação ocupacional dos clientes (Notoh et al., 2014). A avaliaçáo da participação ocupacional é uma chave no processo terapêutico na medida em que informa os aspectos que são essenciais para que uma pessoa possa participar em ocupaçóes, por exemplo, a sua motivação, os seus padrōes de ocupaçóes (rotinas e papéis ocupacionais), as suas habilidades motoras, cognitivas e sociais e em como o ambiente se relaciona para gerar a participaçáo. Assim, medidas válidas e confiáveis são essenciais para orientar intervençóes e pesquisas (Maciver et al., 2016). Também é importante que os pesquisadores e profissionais de outros países sejam capazes de utilizar instrumentos com sensibilidade para medir mudanças e resultados de intervençóes (Coster \& Mancini, 2015). Nessa perspectiva, as intervençóes com pessoas com deficiências devem considerar a complexidade do conceito de participação, envolvendo processos educativos, preventivos, adaptativos e de saúde. 
Particularmente, o MOHO é um modelo que pode ser útil para ações do terapeuta ocupacional no campo da Educação Especial, porque permite identificar a participação em diferentes papéis e os problemas relacionados a essa participação. Por exemplo, um adolescente com deficiência em uma escola pode apresentar problemas ocupacionais como estudante e que precisam de uma compreensão para observarem-se quais são os aspectos que podem ser focados a fim de aumentar a sua participação; se há a necessidade de uma avaliação mais aprofundada sobre como o ambiente escolar pode restringir a participaçáo nos espaços externos, ou se há questóes relacionadas à motivação como interesses em determinadas atividades que facilitariam os processos de ensino e de aprendizagem.

O MOHOST Brasil é uma medida focada na ocupação e centrada no cliente, ou seja, ele permite acessar informaçóes para avaliar e orientar intervençóes e pesquisas em terapia ocupacional (Forsyth et al., 2011). Ele também pode ser usado por terapeutas ocupacionais em conjuntos com outros profissionais para avaliar uma ampla gama de clientes com problemas psicossociais ou deficiências físicas, a fim de compartilhar informaçóes com a equipe de reabilitação (Notoh et al., 2013). Recentemente, esse instrumento foi adaptado transculturalmente para o Brasil e apresentou excelentes evidências de confiabilidade no teste-reteste (Cruz et al., 2019).

O MOHOST tem sido usado como um protocolo de avaliação entre pacientes hospitalizados em uma unidade psiquiátrica aguda localizada no Reino Unido (Parkinson et al., 2008), para avaliar pessoas com dificuldades de aprendizagem e para facilitar consistentemente a prática baseada em evidências (Hawes \& Houlder, 2010).

No que diz respeito às propriedades psicométricas do MOHOST, as evidências atuais mostram que esse instrumento apresenta bons resultados. No Japão, um estudo realizado com 44 pessoas com deficiência física relata boa discriminação no nível de participação ocupacional (Notoh et al., 2013). Já na China, por meio da aplicação da análise de Rash e análise de correlação, um estudo com 101 indivíduos apresentando comprometimentos de ordem mental examinou a validade construtiva, convergente, divergente, concluindo que a versão chinesa é válida para essa população (Pan et al., 2011).

Um estudo realizado na Inglaterra com 1.039 clientes com transtornos psiquiátricos confirma que o MOHOST pode ser usado para investigação científica e propósitos clínicos, pois demonstrou boa validade de construto, confiabilidade e validade concorrente (Kielhofner et al., 2010). Um estudo realizado no Japão examinou o aspecto estrutural da validade do MOHOST Japão, com a Medida de Independência Funcional em 115 pacientes internados em enfermarias de reabilitação. O estudo determinou, com base nos coeficientes de correlação de Spearman, que os dois instrumentos eram altamente correlacionados e se complementavam na avaliação da participação ocupacional (Notoh et al., 2014).

Justifica-se que, embora a literatura examinada traga que o MOHOST já foi traduzido para 19 idiomas, a versão brasileira é a mais recente, adaptada em 2017 por Cruz et al. (2019). Dessa forma, este estudo é o primeiro realizado no Brasil e teve como objetivo verificar se a participação ocupacional medida pelo MOHOST tem correlação com outras medidas de avaliação - nesse caso, ao nível de independência (Medida de Independência Funcional) e a cognição (Mini-Exame do Estado Mental - MEEM) em adultos com deficiências físicas causadas por condições neurológicas. Como objetivos específicos, este estudo buscou examinar a 
validade de constructo da subescala habilidades processuais do MOHOST com os itens cognitivos avaliados na MIF e no MEEM e testar a validade de constructo das habilidades motoras do MOHOST com o domínio motor da MIF.

Esta pesquisa teve como hipótese que a participação ocupacional está associada a um maior nível de independência funcional e de habilidades cognitivas e de que o MOHOST seria sensível para identificar essas relaçóes. Cabe esclarecer que estudos como este são necessários no Brasil, uma vez que muitos instrumentos de avaliação se encontram em língua inglesa. Para além da tradução, os estudos requerem uma adaptação transcultural e, também, uma testagem das propriedades psicométricas do instrumento como, por exemplo, a validade de constructo, para identificar se uma medida mensura aquilo que se propóe (Polit, 2012).

\section{Métodos}

Este estudo foi aprovado pelo Comitê de Ética em Pesquisa com Seres Humanos da Universidade Federal de São Carlos (número de Protocolo 68946717.7.0000.5504) e todos os participantes assinaram o Termo de Consentimento Livre e Esclarecido. Dito isso, esta seção trata do delineamento da pesquisa e dos critérios de inclusão da amostra, dos instrumentos e dos procedimentos que envolveram a coleta de dados e como se deu a análise estatística.

\subsection{Delineamento da PESQUisa E CRITÉrios de INClusáo da AMOSTRA}

Trata-se de um estudo correlacional entre as variáveis de participação ocupacional medidas pelo MOHOST versão 2.0 (Cruz et al., 2019; Parkinson et al., 2006). Uma amostra de conveniência de 41 pessoas com deficiência física foi recrutada em duas unidades de reabilitação localizadas no sudeste do Brasil. A amostra selecionada apresentava diagnósticos neurológicos e deficiências físicas resultantes de lesóes neurológicas. Os critérios de inclusão foram: ter deficiência física; ter sido assistido por um serviço de reabilitação por pelo menos um mês; e ter 18 anos ou mais. Essa amostra é diferente da existente em outros estudos já publicados com a utilização do MOHOST, sendo a maioria deles em pessoas com problemas de saúde mental, o que constitui, dessa forma, um estudo original e pioneiro no Brasil.

Cabe explicar que o MOHOST não é uma medida de avaliação de deficiências, mas de problemas na participaçáo; por essa razão, a pesquisa abrangeu diagnósticos diferenciados, uma vez que o objetivo consistiu em identificar alterações na participação e não vincular esses problemas por diagnósticos específicos, entendendo-se que os problemas ocupacionais resultam de uma compreensão dinâmica sistêmica e esta não pode ser reduzida sob um ponto de vista biomédico.

Para a definição do tamanho da amostra, utilizou-se o cálculo amostral conforme descrito por Walter et al. (1988). Pelo cálculo recomendado, na análise da MIF, teríamos a necessidade de 34 sujeitos para alcançar um poder de estimativa de $99 \%$, considerando alfa $=0,05$, para uma correlação aceitável de 0,70, tendo alcançado um índice de correlação de 0,46. Aplicando o mesmo cálculo para os valores observados no MEEM, com 42 sujeitos, alcançou-se um poder de estimativa de $95 \%$, considerando os mesmos parâmetros para um índice de 
correção de 0,52 . Por esse motivo, acredita-se que a associação observada revela um fenômeno correlacional.

\subsection{INSTRUMENTOS}

Três instrumentos foram usados para a coleta de dados: 1) Instrumento de Identificação da Participação Ocupacional (MOHOST Brasil 2.0); 2) Mini-Exame do Estado Mental (MEEM); e 3) Medida de Independência Funcional (MIF). Esses instrumentos foram aplicados pelos mesmos terapeutas, mas estes desconheciam os objetivos da pesquisa.

Esses instrumentos foram validados para o Brasil e são medidas padronizadas com escores que avaliam constructos semelhantes aos abordados por alguns dos fatores do MOHOST, por exemplo: habilidades motoras e cognitivas. A escolha desses instrumentos, em particular, também foi baseada no fato de que o constructo do MOHOST é a participação ocupacional em Atividades da Vida Diária (AVD), e a MIF é uma medida centrada em AVD e que apresenta os constructos motor e cognitivo que influenciam no desempenho de determinadas ocupaçóes. Para o MEEM, considerou-se o constructo da cognição identificado no item de habilidades processuais do MOHOST, o que possibilitaria testar uma validade de constructo, bastante utilizada em outros estudos (Mattos et al., 2011; Notoh et al., 2014). Os instrumentos são brevemente descritos a seguir.

1. Instrumento de Identificação da Participação Ocupacional (MOHOST Brasil 2.0): trata-se de uma avaliação observacional da participaçáo ocupacional utilizada por terapeutas os quais avaliam até que ponto os clientes estão envolvidos em ocupaçóes (Lee et al., 2013; Maciver et al., 2016). O MOHOST é dividido em seis fatores: motivação para ocupação (avaliação de capacidade, expectativa de sucesso, interesse e escolhas), padrão de ocupação (rotina, adaptabilidade, papéis, responsabilidades), habilidades de comunicação e interação (habilidades não verbais, conversação, expressão vocal, relacionamentos), habilidades de processo (conhecimento, tempo, organização, resolução de problemas), habilidades motoras (postura e mobilidade, coordenação, força e esforço, energia) e meio ambiente (espaço físico, recursos físicos, grupos sociais, demandas ocupacionais) (Parkinson et al., 2006). Os critérios usados para classificar cada fator são: F = facilita a participação ocupacional; $\mathrm{P}$ = permite a participação ocupacional; $\mathrm{I}$ = inibe a participaçáo ocupacional; e $\mathrm{R}$ = restringe a participação ocupacional (Parkinson et al., 2006). Essas letras são transformadas em pontuaçóes com base em uma escala de quatro pontos em que $\mathrm{F}=4, \mathrm{P}=3, \mathrm{I}=2$ e $\mathrm{R}=1$ (Kramer et al., 2009; Parkinson et al., 2006). A pontuação total obtida para os 24 itens varia de um mínimo de 24 pontos a 96 pontos e a aplicação leva de 30 a 60 minutos (Notoh et al., 2013; Pan et al., 2011).

2. Mini-Exame do Estado Mental (MEEM): esse instrumento é amplamente utilizado para rastreio cognitivo e apresenta propriedades psicométricas de validade para a população brasileira (Caro et al., 2016; Lourenço \& Veras, 2006; Melo et al., 2017). O MEEM é dividido em seis itens: orientação, registro, atenção e cálculo, evocação, linguagem e práxis, cópia, com pontuação máxima de 30 pontos. Essa medida foi escolhida para testar se existiam associaçóes com o construto da cognição, também presente no fator "Habilidades do 
Processo" do MOHOST (Parkinson et al., 2006). Esse instrumento também foi utilizado em um estudo anterior de validação do MOHOST (Kielhofner et al., 2010).

3. Medida de Independência Funcional (MIF): O objetivo desse instrumento é avaliar o nível de independência funcional nas atividades da vida diária (AVD), subdivididas em subescalas motora e cognitiva. A subescala motora inclui: autocuidado, mobilidade, controle de esfíncter, transferência, locomoção. A subescala de cognição inclui comunicação e cognição social. A MIF é composta por 18 itens que classificam a independência funcional como Independente (7 pontos para independência completa e 6 pontos para independência modificada); Dependência Modificada (Supervisão - 5 pontos, Assistência Mínima - 4 pontos e Assistência Moderada - 3 pontos) e Dependência Completa (Assistência Máxima - 2 pontos e Assistência Total - 1 ponto). A pontuação total varia de 18 a 126 pontos, enquanto a pontuação total da subescala motora está entre 13 e 91 pontos. A pontuação para a subescala cognitiva está entre 5 e 35 pontos, o que apresentou validade e confiabilidade para a população brasileira (Riberto et al., 2004). Esse instrumento foi escolhido para verificar a existência de correlações do MOHOST comparando as subescalas motora e cognitiva da MIF com os fatores "Habilidades Motoras" e "Habilidades do Processo" do MOHOST, respectivamente. Além disso, foi levado em consideração o fato de que a MIF mede o nível de independência funcional para realizar AVD, uma ocupação que também é abordada na avaliação MOHOST, tendo em vista que o desempenho das AVD no MOHOST pode ser entendido a partir dos seus domínios de volição, habituação, capacidade de desempenho e ambiente (Notoh et al., 2014). A MIF também foi usada em um estudo anterior, abordando os aspectos estruturais do MOHOST de validade de construto (Notoh et al., 2014).

\subsection{Procedimentos de coleta de dados}

Este estudo foi desenvolvido em duas universidades públicas localizadas no estado de São Paulo, Brasil. O local do estudo incluiu duas unidades de cuidados em saúde focadas na reabilitação de pessoas com deficiências físicas localizadas no Sul e no Sudeste do Brasil. O MOHOST foi aplicado por dez estudantes de Graduação em Terapia Ocupacional e dois professores supervisores. O instrumento foi preenchido a partir do acompanhamento dos pacientes nos serviços de reabilitação. Os aplicadores receberam um treinamento no Modelo da Ocupação Humana, fornecido pelo autor principal, juntamente ao formulário MOHOST contendo instruções.

\subsection{ANÁLise ESTATÍSTICA}

Três variáveis foram utilizadas para caracterizar a amostra (idade, sexo e diagnóstico). Como a idade é uma variável quantitativa, ela foi descrita usando média e desvio padrão. As duas variáveis nominais (sexo e diagnóstico) foram descritas utilizando-se sua frequência relativa em termos de porcentagem da amostra total $(n=41)$.

Análises de correlação foram realizadas para identificar associações da pontuação total do MOHOST com as pontuaçóes geradas pela MIF e MEEM. Nesse caso, as variáveis 
apresentaram um padrão de distribuição normal dos dados, motivo pelo qual se aplicou o teste de correlação de Pearson.

Por fim, também se encontrou associação entre os itens do MOHOST e as pontuaçóes obtidas nas subescalas motora e cognitiva da MIF e entre os itens do MOHOST com os itens do MEEM. Nesse caso, aplicou-se um teste não paramétrico (teste de correlação de Spearman), pois os dados não apresentaram distribuição normal, seguindo a mesma classificação para o coeficiente de correlação $(r)$.

\section{Resultados}

A Tabela 1 descreve a amostra do estudo: uma população com condiçóes de saúde determinadas por comprometimentos do sistema nervoso. A amostra é predominantemente composta por indivíduos adultos do sexo masculino e, com mais frequência, teve-se o diagnóstico de acidente vascular cerebral, que afetou mais da metade da amostra.

\section{Tabela 1}

Caracteristicas da amostra $(N=41)$

\begin{tabular}{llc}
\hline \multicolumn{1}{c}{$\begin{array}{c}\text { Características } \\
\text { Quantitativa/Qualitativa Variáveis* }\end{array}$} & \multicolumn{1}{c}{ Unidades/Classes } & $\begin{array}{c}\text { Amostra } \\
(\mathbf{n}=\mathbf{4 1})\end{array}$ \\
\hline Idade & anos & $51.44 \pm 17.23$ \\
\hline \multirow{2}{*}{ Sexo } & masculino & 25 \\
& feminino & 16 \\
\hline & Acidente vascular encefálico & 22 \\
\cline { 2 - 3 } & Lesão medular & 8 \\
& Doença de Parkinson & 3 \\
& Traumatismo crânio-encefálico & 1 \\
& Ataxia cerebelar & 1 \\
& Aneurisma cerebral & 1 \\
& Tumor cerebral & 1 \\
& Charcot Marie Tooth & 1 \\
& Neuropatia diabética & 1 \\
& Esclerose múltipla & 1 \\
& Neurotoxoplasmose & 1 \\
\hline
\end{tabular}

*Nota. A variável quantitativa é descrita por média \pm desvio padrão. As variáveis qualitativas (nominais) são descritas pela distribuição de frequência relativa como uma porcentagem da amostra total.

A Figura 1 apresenta as correlações analisadas para toda a amostra $(n=41)$ e por meio de associaçóes entre os escores obtidos no MOHOST e os obtidos na MIF e no MEEM. Foi encontrada uma correlação significativa e diretamente proporcional. Foi detectada uma correlação moderada $(0,700 \geq \mathrm{r}>0,500)$ entre o MOHOST e o MEEM. Embora tenha sido 
encontrada uma baixa correlação $(0,500 \geq \mathrm{r}>0,250)$ entre o MOHOST e a MIF, a associação ainda foi significativa $(\mathrm{p}<0,05)$. Esses escores gerais dos três instrumentos contemplam uma análise que informa a correlação entre a participação ocupacional com a independência e cognição, respondendo ao objetivo geral e hipóteses desta pesquisa.

\section{Figura 1}

Correlaçóes entre os instrumentos Medida de Independência Funcional (MIF), Mini-Exame do Estado Mental (MEEM) e MOHOST Brasil

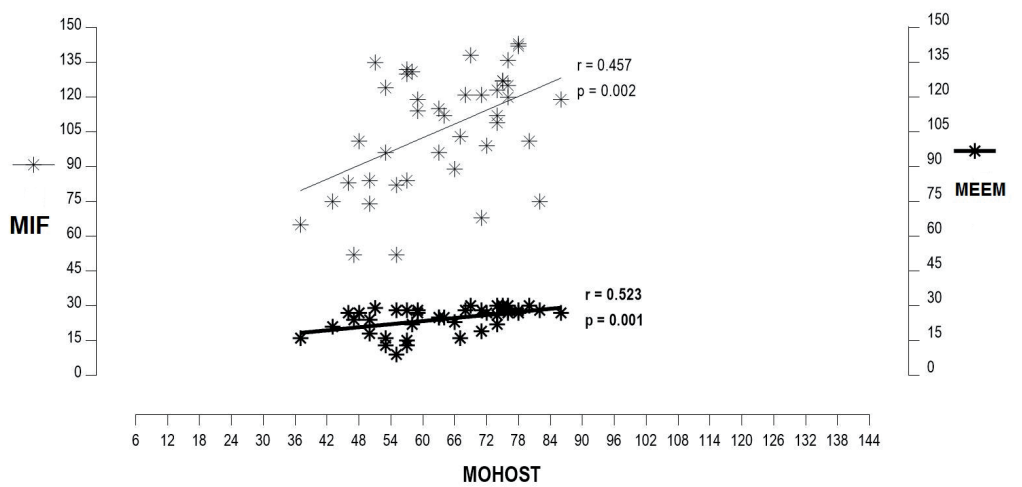

A Tabela 2 apresenta correlações entre MOHOST, MIF e MEEM. Considerando que há uma correlação entre as pontuaçóes obtidas para os fatores do MOHOST com as subescalas motoras e cognitivas da MIF e os itens do MEEM, várias associaçóes significativas foram encontradas entre praticamente todos os fatores do MOHOST e quase todos os itens do MEEM.

A mesma análise, realizada com a MIF, mostrou correlação significativa em apenas dois fatores MOHOST (padrão de ocupação e habilidades motoras) com a subescala motora e um fator MOHOST "Habilidades de comunicação e interação" com a subescala cognitiva da MIF. Não foi encontrada correlação significativa com o fator MOHOST "Ambiente".

As correlações mais altas, duas correlações moderadas $(0,700 \geq \mathrm{r}>0,500)$, foram encontradas entre o fator MOHOST "Habilidades processuais" e o item "Linguagem e Práxis" do MEEM, bem como entre as habilidades motoras do MOHOST e a subescala motora da MIF. Todas as demais correlaçóes, embora significativas ( $p<0,05)$, foram classificadas com força de correlação baixa $(0,500 \geq r \geq 0,250)$, enquanto uma foi classificada com força de correlação pobre ( $r<0,250)$ : a correlação entre o fator MOHOST "Habilidades de comunicação e interação" e a subescala cognitiva da MIF. 


\section{Tabela 2}

Correlação das pontuaçóes por fatores do MOHOST com as pontuaçóes por itens da MIF e MEEM $(N=41)$

\begin{tabular}{|c|c|c|c|c|c|c|c|}
\hline \multicolumn{2}{|c|}{ Correlaçóes* } & \multicolumn{6}{|c|}{ FATORES DO MOHOST } \\
\hline \multicolumn{2}{|c|}{ Spearman r (p valor) } & $\begin{array}{c}\text { Motivação para } \\
\text { ocupação }\end{array}$ & $\begin{array}{l}\text { Padrão de } \\
\text { ocupaçáo }\end{array}$ & $\begin{array}{c}\text { Habilidades de } \\
\text { comunicação e } \\
\text { interaçấo }\end{array}$ & $\begin{array}{c}\text { Habilidades } \\
\text { processuais }\end{array}$ & $\begin{array}{l}\text { Habilidades } \\
\text { motoras }\end{array}$ & Ambiente \\
\hline \multirow[b]{2}{*}{ MIF } & $\begin{array}{l}\text { Subescala } \\
\text { motora }\end{array}$ & $0.237(0.135)$ & $0.376(0.015)$ & $0.123(0.442)$ & $0.326(0.037)$ & $0.586(0.001)$ & $0.277(0.080)$ \\
\hline & $\begin{array}{l}\text { Subescala } \\
\text { cognitive }\end{array}$ & $0.046(0.101)$ & $-0.047(0.872)$ & $0.243(0.001)$ & $0.079(0.179)$ & $0.046(0.776)$ & $-0.047(0.769)$ \\
\hline \multirow{6}{*}{ MEEM } & Orientação & $0.252(0.111)$ & $0.155(0.333)$ & $0.436(0.004)$ & $0.181(0.257)$ & $0.045(0.779)$ & $0.204(0.199)$ \\
\hline & Registro & $0.256(0.106)$ & $0.049(0.762)$ & $0.236(0.136)$ & $0.001(0.994)$ & $-0.256(0.107)$ & $0.070(0.662)$ \\
\hline & $\begin{array}{l}\text { Atenção e } \\
\text { cálculo }\end{array}$ & $0.170(0.289)$ & $0.120(0.453)$ & $0.320(0.041)$ & $0.221(0.165)$ & $0.198(0.215)$ & $0.180(0.261)$ \\
\hline & Evocaçáo & $0.420(0.006)$ & $0.231(0.146)$ & $0.440(0.003)$ & $0.326(0.037)$ & $0.111(0.490)$ & $0.162(0.310)$ \\
\hline & $\begin{array}{l}\text { Linguagem } \\
\text { e Práxis }\end{array}$ & $0.386(0.012)$ & $0.371(0.016)$ & $0.447(0.003)$ & $0.573(0.001)$ & $0.356(0.004)$ & $0.417(0.058)$ \\
\hline & Cópia & $0.392(0.011)$ & $0.132(0.411)$ & $0.298(0.058)$ & $0.381(0.014)$ & $0.318(0.043)$ & $0.284(0.072)$ \\
\hline
\end{tabular}

${ }^{*}$ Nota. Os valores de Spearman r e p valor entre cada par de itens foram distribuídos na Tabela. Células em destaque indicam correlaçóes significativas $(\mathrm{p}<0.05)$.

\section{Discussáo}

Terapeutas ocupacionais buscam medidas confiáveis para serem usadas na prática clínica, pois há uma demanda crescente por práticas baseadas em evidências, o que pode ser facilitado usando avaliaçóes padronizadas como o MOHOST (Hawes \& Houlder, 2010).

A análise das pontuações gerais obtidas para o MOHOST, MIF e MEEM apresentaram associação entre essas medidas, pois mostra como as diferentes dimensões do fazer estão relacionadas, respectivamente, à participação, ao desempenho e às habilidades (De Las Heras De Pablo et al., 2017). Particularmente, a relação identificada neste estudo entre a MIF e o MOHOST é semelhante às descobertas de um estudo anterior que abordou a validade de construto do MOHOST. A validade do construto para o MOHOST-J (Japão) é confirmada usando outro tipo de análise (modelo Rasch e análise fatorial confirmatória). Os dados obtidos de 115 clientes japoneses em ambientes de reabilitação indicam que o nível de correlação entre o MOHOST e a MIF foi eficaz para avaliar o nível de engajamento dos clientes nas AVD e medir a participação ocupacional (Notoh et al., 2014).

Também foi identificada uma associação semelhante à encontrada neste estudo em relação ao MOHOST e às AVD, em uma pesquisa que examinou as propriedades psicométricas do MOHOST com a Escala de Resultados de Saúde da Nação (HoNOS), com uma amostra de 1.039 pessoas com problemas psiquiátricos. Os resultados mostram que os fatores do MOHOST apresentam validade convergente com as AVD medidas pelo HoNOS, enquanto o "padrão de ocupação" e a "motivação para a ocupação" se destacaram (Kielhofner et al., 2010). Essas associaçóes entre participação ocupacional e AVD são explicadas pelo fato de o MOHOST ter sido projetado para detectar problemas que interferem nas AVD; entendendo-se por autocuidado produtividade e lazer (Parkinson et al., 2006). 
A correlação encontrada entre a subescala motora da MIF e o fator "Habilidades Motoras" do MOHOST era esperada, porque a amostra deste estudo apresenta deficiências físicas que interferem nessas habilidades ocupacionais, mostrando a coesão entre as medidas de ambos os instrumentos. Um estudo realizado no Japão aplicou o MOHOST com 44 participantes com diversas deficiências físicas. Foi identificado que a maior parte da amostra apresentou dificuldades dentro do fator "Habilidades Motoras", relacionadas à "postura e mobilidade" e "coordenação" (Notoh et al., 2013).

Destaca-se uma relação interessante, encontrada neste estudo, entre a subescala motora da MIF e o "Padrão de ocupação" do MOHOST. Esse resultado apoia uma discussão sobre como as alteraçóes motoras decorrentes de uma deficiência física afetam o desempenho ocupacional de um indivíduo nas AVD. Isso é explicado pelo fator "padrão de ocupação" ser a "habituação" no Modelo de Ocupação Humana, conceito que abrange os hábitos e papéis ocupacionais (Lee \& Kielhofner, 2017). Esses papéis ocupacionais podem estar comprometidos em pessoas com deficiências físicas (Cruz \& Emmel, 2013) e, consequentemente, impactar no seu desempenho e participação nas AVD, considerando que a participação em papéis ocupacionais informa ambos: o desempenho e a participação ocupacional (Bonsaksen et al., 2015).

Por outro lado, os resultados também sustentam a discussão de um aspecto-chave do Modelo de Ocupação Humana, que reconhece que limitaçóes e restrições à participação ocupacional podem não ser uma consequência direta de deficiências, mas o resultado de influências multifacetadas entre os componentes do modelo (Maciver et al., 2016), quando se considera, por exemplo, os interesses e os valores da pessoa e a sua relação com hábitos e papéis.

As várias correlaçóes encontradas, neste estudo, entre os itens do MEEM com os fatores do MOHOST mostram uma associação entre os componentes objetivos da capacidade de desempenho que envolvem a participação ocupacional. Essa capacidade de desempenho envolve não apenas os componentes musculoesqueléticos, neurológicos ou cardiopulmonares, mas também depende de habilidades cognitivas, como a memória, uma vez que, quando as pessoas realizam tarefas, elas exercem tais capacidades (Tham et al., 2017).

A associação encontrada, nesta pesquisa, entre a linguagem e a práxis do MEEM com todos os fatores do MOHOST, com exceção do fator "Ambiente", pode ser explicada pelo fato de que o repertório cognitivo é uma habilidade relacionada ao indivíduo. Eles foram correlacionados com todos os fatores individuais do MOHOST porque a linguagem é uma habilidade necessária para a interação na maioria dos desempenhos ocupacionais. Além disso, o fator do MOHOST de "Habilidades de Comunicação e Interação" foi correlacionado com a subescala cognitiva da MIF e com quatro dos seis itens que compóem o MEEM, mostrando associação entre cognição e linguagem, uma vez que são habilidades relacionadas à participação ocupacional. A multiplicidade de tarefas exige que as pessoas interpretem o mundo para planejar, comunicar-se e interagir com ele (Tham et al., 2017).

O estudo realizado por Pan et al. (2011), que investigou a validade convergente da versão chinesa do MOHOST em uma amostra de 101 indivíduos com problemas de saúde mental, relata resultados semelhantes aos encontrados em nosso estudo. Os autores verificaram que o MEEM estava moderadamente correlacionado aos fatores "habilidades processuais" $\mathrm{e}$ fortemente correlacionado ao fator "habilidades de comunicação e interação" quando estas 
foram avaliadas. Os dados deste estudo em relação à linguagem e à cogniçãa também foram apoiados por um estudo anterior que mostra uma relação entre os fatores "habilidades de comunicação e interação" e "habilidades processuais" com os itens do HoNOS "problemas com relacionamentos" e "problemas cognitivos" (Kielhofner et al., 2010).

Um resultado inesperado, no entanto, foi a ausência de correlação entre a MIF, o MEEM e o fator "Ambiente" do MOHOST. Uma hipótese é que esse resultado se deve pelo fato de todos os instrumentos utilizados nesta pesquisa se concentram nos aspectos individuais dos clientes e não nos aspectos ambientais. Outra possível explicação é que o MOHOST não avalia o ambiente em si, mas a forma com que o ambiente apoia a participação ocupacional. Tal resultado sugere a necessidade de mais estudos empregando uma combinação de métodos e instrumentos para obter informaçóes sobre o meio ambiente e seus componentes físicos, sociais e ocupacionais (Fisher et al., 2017).

\section{Conclusótes}

Este estudo identificou correlaçóes entre a participação ocupacional e a independência funcional para as AVD e habilidades cognitivas, necessárias para a aprendizagem de atividades que informam a participação de pessoas com deficiências físicas. Com exceção do domínio "Ambiente", pode-se afirmar que, a partir da identificação de correlações gerais entre o MOHOST e a MIF e entre o MOHOST e o MEEM é possível concluir, há relação entre as dimensões de participação, desempenho e habilidades, confirmando que o MOHOST é útil para avaliar a participação ocupacional de pessoas com deficiências físicas.

Este estudo apresenta limitações, sendo elas: a inexistência de outros instrumentos validados e que medem a participação ocupacional no Brasil, os quais, de certa forma, restringiram, por exemplo, testar a validade convergente aos itens dos instrumentos selecionados para esta pesquisa; uma amostra de conveniência não permite que os resultados sejam generalizados para todos aqueles com deficiência física. Reconhece-se que o desenho deste estudo, baseado em relaçóes de correlação, pode dar seguimento a estudos futuros, por exemplo, com análises como a Rasch.

No entanto, cabe destacar como peculiaridade que este é um estudo inédito, pois se trata da primeira pesquisa conduzida no Brasil, cujo foco é a participação ocupacional de pessoas com deficiências utilizando uma medida adaptada transculturalmente e com excelente confiabilidade. Além disso, os dados apresentados podem direcionar futuros estudos comparativos que possam ser desenvolvidos para conhecer aspectos da participação de pessoas com deficiências em diferentes ambientes, como casa, trabalho, escola, espaços de lazer.

Por fim, o instrumento MOHOST pode ser útil para informar a participação ocupacional de pessoas e para fornecer dados que possam contribuir para o planejamento de intervençôes que estimulem a participação ocupacional em diferentes papéis no âmbito da Terapia Ocupacional e na sua inferface com a Educação Especial. 


\section{REFERÊNCIAS}

Bonsaksen, T., Meidert, U., Schuman, D., Kvarsnes, H., Haglund, L., Prior, S., Forsyth, K., Yamada, T., \& Scott, P. (2015). Does the role checklist measure occupational participation? The Open J Occup Ther, 3(3), 1-12. https://doi.org/10.15453/2168-6408.1175

Caro, C. C., Mendes, P. V. B., Costa, J. D., Nock, L. J., \& Cruz, D. M. C. (2016). Independence and cognition post-stroke and its relationship to burden and quality of life of family caregivers. Topics in stroke rehabilitation, 24(3), 194-199. https://doi.org/10.1080/10749357.2016.1234224

Coster, W. J., \& Mancini, M. C. (2015). Recommendations for translation and cross-cultural adaptation of instruments for occupational therapy research and practice. Rev Ter Ocup USP, 26(1), 50-57. http://doi.org/10.11606/issn.2238-6149.v26ilp50-7

Cruz, D. M. C., \& Emmel, M. L. G. (2013). Associations among occupational roles, independence, assistive technology, and purchasing power of individuals with physical disabilities. Revista LatinoAmericana de Enfermagem, 21(2), 484-491. https://doi.org/10.1590/S0104-11692013000200003

Cruz, D. M. C., Parkinson, S., Rodrigues, D. S., Carrijo, D. C. M., Costa, J. D., Fachin-Martins, E., \& Pfeifer, L. I. (2019). Cross-cultural adaptation, face validity and reliability of the Model of Human Occupation Screening Tool to Brazilian Portuguese. Cad Bras Ter Ocup, 27(4), 691-702. https:// doi.org/10.4322/2526-8910.ctoao2007

De Las Heras De Pablo, C. G., Wei-Fan, C., \& Kielhofner, G. (2017). Dimensions of doing. In R. Taylor (Ed.), Kielhofner's Model of Human Occupation (pp. 107-122). Wolters Kluwer.

Fisher, G., Parkinson, S., \& Haglund, L. (2017). The environment and human occupation. In R. Taylor (Ed.), Kielhofner's Model of Human Occupation: theory and application (pp. 91-106). Wolters Kluwer.

Forsyth, K., Parkinson, S., Kielhofner, G., Kramer, J., Mann, L. S., \& Duncan, E. (2011). The measurement properties of the model of human occupation screening tool and implications for practice. New Zealand J Occup Ther, 58(2), 5-13.

Hawes, D., \& Houlder, D. (2010). Reflections on using the Model of Human Occupation Screening Tool in a joint learning disability team. British J Occup Ther, 73(11), 564-567. https://doi.org/10. 4276/030802210X12892992239431

Kielhofner, G., Fan, W., Morley, M., Garnham, M., Heasman, D., Forsyth, K., Lee, S., \& Taylor, R. (2010). Psychometric study of the Model of Human Occupation Screening Tool (MOHOST). Hong Kong J Occup Ther, 20(2), 63-70. https://doi.org/10.1016/S1569-18611170005-5

Kramer, J., Kielhofner, G., Lee, S. W., Ashpole, E., \& Castle, L. (2009). Utility of the Model of Human Occupation Screening Tool for detecting client change. Occup Ther in Mental Health, 25(2), 181191. https://doi.org/10.1080/01642120902859261

Lee, S. W., Forsyth, K., Morley, M., Garnham, M., Heasman, D., \& Taylor, R. R. (2013). Mental health payment-by-results clusters and the Model of Human Occupation Screening Tool. OTJR: Occupation, Participation and Health, 33(1), 40-49. https://doi.org/10.3928/1539449220120426-01

Lee, S. W., \& Kielhofner, G. (2017). Habituation: patterns of daily occupation. In R. Taylor (Ed.), Kielhofner's Model of Human Occupation (pp. 57-73). Wolters Kluwer.

Lourenço, R. A., \& Veras, R. P. (2006). Mini-Mental State Examination: psychometric characteristics in elderly outpatients. Rev Saúde Pública, 40(4), 712-719. https://doi.org/10.1590/S003489102006000500023

Maciver, D., Morley, M., Forsyth, K., Bertram, N., Edwards, T., Heasman, D., Rennison, J., Rush, R., \& Willis, S. (2016). A Rasch analysis of the model of human occupation screening tool single 
observation form (MOHOST-SOF) in mental health. British J Occup Therapy, 79(1), 49-56. https://doi.org/10.1177/0308022615591173

Mattos, P., Segenreich, D., Dias, G. M., Nazar, B., Saboya, E., \& Broad, M. (2011). Validade de constructo e confiabilidade da versão em língua portuguesa do Questionário de Qualidade de Vida em Adultos que apresentam TDAH (AAQoL). Rev. Psiquiatr. Clín., 38(3), 91-96. https://doi. org/10.1590/S0101-60832011000300002

Melo, D. M., Barbosa, A. J. G., \& Neri, A. L. (2017). Mini-mental State Examination: Validity evidence based on internal structure. Avaliação Psicológica, 16(2), 161-168. http://doi.org/10.15689/ AP.2017.1602.06

Notoh, H., Yamada, T., Kobayashi, N., Ishii, Y., \& Forsyth, K. (2013). Examining the psychometric properties of the model of human occupation screening tool-Japanese version. Hong Kong J Occup Ther, 23(1), 26-31. https://doi.org/10.1016/j.hkjot.2013.06.001

Notoh, H., Yamada, T., Kobayashi, N., Ishii, Y., \& Forsyth, K. (2014). Examine the structural aspect of the construct validity of the Japanese version of the Model of Human Occupation Screening Tool. British J Occup Therapy, 77(10), 516-525. https://doi.org/10.4276/030802214X14122630932476

Pan, A., Fan, C., Chung, L., Chen, T., Kielhofner, G., Wu, M., \& Chen, Y. (2011). Examining the validity of the Model of Human Occupation Screening Tool: Using classical test theory and item response theory. British J Occup Ther, 74(1), 34-40. https://doi.org/10.4276/03080221 $1 \mathrm{X} 12947686093648$

Parkinson, S., Forsyth, K., \& Kielhofner, G. (2006). User's manual for the Model of Human Occupation Screening Tool (MOHOST) Version 2.0. University of Illinois.

Parkinson, S., Chester, A., Cratchley, S., \& Rowbottom, J. (2008). Application of the Model of Human Occupation Screening Tool (MOHOST Assessment) in an Acute Psychiatric Setting. Occup Ther Health Care, 22(2-3),63-75. https://doi.org/10.1080/07380570801989465

Polit, D. F., \& Beck, C. T. (2012). Nursing research: generating and assessing evidence for nursing practice. Wolters Klower Health, Lippincott Williams \& Wilkins.

Riberto, M., Miyazaki, M. H., Jucá, S. S. H., Sakamoto, H., Pinto, P. P. N., \& Battistella, L. R. (2004). Validation of the Brazilian version of Functional Independence Measure. Acta Fisiatr, 11(2), 72-6. https://doi.org/10.5935/0104-7795.20040003

Tham, K., Erikson, A., Fallaphour, M., Taylor, R., \& Kielhofner, G. (2017). Performance capacity and the lived body. In R. Taylor (Ed.), Kielhofner's Model of Human Occupation (pp. 74-90). Wolters Kluwer.

Vessby, K., \& Kjellberg, A. (2010). Participation in occupational therapy research: a literature review. British J Occup Therapy, 73(7), 319-326. https://doi.org/10.4276/030802210X12759925544380

Walter, S. D., Eliasziw, M., \& Donner, A. (1998). Sample size and optimal designs for reliability studies. Statistics in Medicine, 17, 101-100. https://doi.org/10.1002/(SICI)10970258(19980115)17:1<101::AID-SIM727>3.0.CO;2-E

Recebido em: 03/08/2020

Reformulado em: 10/09/2020

Aprovado em: 17/10/2020 
CRUZ, D.M.C. et al. 